\title{
Parry-Romberg syndrome: a case with a possible association with Lyme disease
}

\author{
Nicola di Meo ${ }^{1}$, Giuseppe Stinco², Katiuscia Nan ${ }^{1 凶}$, Caterina Pinzani², Giusto Trevisan ${ }^{1}$
}

\begin{abstract}
Parry-Romberg syndrome is an acquired slowly progressive disease characterized by an atrophy mostly involving half of the face. The pathogenesis of this disfiguring condition is still controversial. The relationship between Parry-Romberg syndrome and Lyme disease needs to be considered in depth. A 16-year-old woman from Albania presented with linear depressions of the right side of the face, clinically compatible with Parry-Romberg syndrome. She had a positive history of Lyme disease. Borrelia infection was confirmed by the positivity of PCR and the presence of IgM antibodies. The patient received intravenous penicillin and metronidazole for 14 days. After treatment and during a 2-year follow-up, the clinical disease progression was halted and the serological and microbiological tests for Borrelia burgdorferi sensu lato were negative. We cannot exclude a coincidence, however, of the bacteriological and serological evidence. Moreover, the interruption of the disease progression after the antibiotic therapy is difficult to ignore without claiming that this association is at least suggestive.
\end{abstract}

Keywords: Parry-Romberg, Lyme disease, borreliosis, Borrelia, morphea

Received: 13 May 2015| Returned for modification: 16 July 2015 | Accepted: 20 August 2015

\section{Introduction}

Parry-Romberg syndrome is an uncommon acquired slowly progressive hemifacial atrophy of unknown etiology that also rarely involves the ipsilateral part of the limbs (1). This syndrome has many features of linear scleroderma "en coup de sabre," but it is characterized by more extensive involvement of the lower face with only a slight cutaneous sclerosis (2). It is due to gradual wasting of the subcutaneous fat, accompanied by an atrophy of the skin and, infrequently, of the muscles and bones. In particular, bone and cartilage tissues are rarely affected, unless the onset occurs before the second decade, when the face and skull structures are not fully developed (2). The average onset of this disease is in the first two decades, beginning with a progressive phase that may span up to 20 years and thereafter tending toward stabilization (1). In $15 \%$ of the cases, neurological signs and symptoms such as trigeminal neuralgia, facial paresthesia, headache, and focal epilepsy are present (1). The pathogenesis of this syndrome remains unexplained: it is thought to be a unilateral inflammatory process associated with a chronic vascular or neurogenic disturbance such as sympathetic dysfunction (3). Other postulated causes include autoimmunity, inheritance, local trauma, focal scleroderma, endocrine and metabolic disorders, and other factors, including Borrelia burgdorferi sensu lato infections (1-4). The role of Borrelia burgdorferi sensu lato in the development of atrophosclerodermic diseases is well known even when the facial area is involved (5-7). In the literature there are few reports showing the association between Parry-Romberg syndrome and borreliosis (2, 8-11). We present another case of Parry-Romberg syndrome in which the correlation with Borrelia burgdorferi infection could be strongly suspected.

\section{Case report}

A 16-year-old woman from Albania presented with linear depres- sions of the right side of the face, clinically compatible with Parry-Romberg syndrome. She developed a progressive facial right asymmetry from the age of 11 and had no memory of any significant local trauma and no family history of progressive hemifacial atrophy or similar conditions. Dermatological examination revealed a linear atrophic depression of soft tissues, especially of the lower part of the right cheek and of the right side of the chin with labial asymmetry and tongue hemiatrophy. No eyebrow or forehead alopecia was present (Fig 1). There was no evidence of sensory or motor functional deficits on either side. Lyme ELISA (NovaTec Immunodiagnostica $\mathrm{GmbH}$ ) IgM antibodies (antigens: Flagellin recombinant and purified OspC) were elevated in serum (178 UA/ml, normal values < $24 \mathrm{UA} / \mathrm{ml}$ ), whereas IgG were normal. The IgM positivity was confirmed by immunoblot test (NovaTec Immunodiagnostica $\mathrm{GmbH}): 24 \mathrm{kDA}(\mathrm{OspC}), 41 \mathrm{kDA}(\mathrm{Fla})$, $39 \mathrm{kDA}(\mathrm{p} 39)$. The patient remembered a tick bite 5 to 6 years earlier in the upper right area of the neck, close to the subsequent atrophic area, with a history of a retarded surrounding erythema, spontaneously resolved, most likely attributable to an erythema chronicum migrans. At that time she was not tested. A complete blood cell count, chemistry panel, and lipid profile revealed unremarkable findings. Serum and urine protein electrophoresis tests produced normal results, as did liver-, renal-, and thyroidfunction tests, erythrocyte sedimentation rate, $\mathrm{C}$-reactive protein level, antinuclear antibodies, human immunodeficiency virus antibodies, hepatitis B and C surface antigens, and VDRL test. Only a low count of $\mathrm{CD} 57+$ natural killer cells was present. Polymerase chain reaction real time (RT-PCR) assay for Borrelia burgdorferi sensu lato detection, performed on DNA obtained from peripheral blood, was positive. The patient refused a new biopsy on her face in order to perform a culture of Borrelia burgorferi sensu lato. All subcutaneous structures were intact: an X-ray of the facial mass showed that the two halves of the facial skeleton were symmetrical and regularly developed. No consistent central and peripheral nervous system disorder was noticed, neither based on patient

${ }^{1}$ Dermatology and Venereology Department, University of Trieste, Ospedale Maggiore di Trieste, Trieste, Italy. ${ }^{2}$ Department of Clinical and Experimental Pathology and Medicine, Institute of Dermatology, University of Udine, Academic Hospital of Udine, Udine, Italy. $\bowtie$ Corresponding author: katiuscia. nan@gmail.com 
history or physical and electrographic examination (EEG), nor based on the quality of cerebrospinal fluid (colorless, with a negative Pandy reaction and with glucose, electrolytes, and proteins in the normal range) and on neuroimaging (brain and brainstem SPECT and MRI). In line with serological positivity for Lyme disease, the patient underwent an intravenous antibiotic treatment with intravenous penicillin $\mathrm{G}$ at a dosage of 20 million units per day associated with intravenous metronidazole ( $500 \mathrm{mg}$ daily) for 14 days. After 6 months, the IgM antibodies were $89 \mathrm{UA} / \mathrm{ml}$, with the same immunoblot panel and blood PCR negative. After 14 months, the IgM antibodies were $36 \mathrm{UA} / \mathrm{ml}$, the IgM immunoblot was positive for p39 and p41, and blood PCR was negative. After 24 months, the clinical disease progression had definitely halted and IgM antibodies as well as blood PCR for Borrelia burgdorferi sensu lato were negative. In order to correct the residual facial asymmetry and reshape the facial contour, a lipostructure session was carried out (Fig. 2).

\section{Discussion}

Since the first description of Parry-Romberg syndrome in 1825 , this syndrome has aroused questions and reflection about its pathogenesis. It is not yet well known and seems to be heterogeneous, including trauma, infection, heredity, vascular malformation, auto-immunity, endocrine disturbances, disturbance of fat metabolism, and sympathetic dysfunction (12). Although the relationship of Borrelia burgdorferi sensu lato infection to Par-

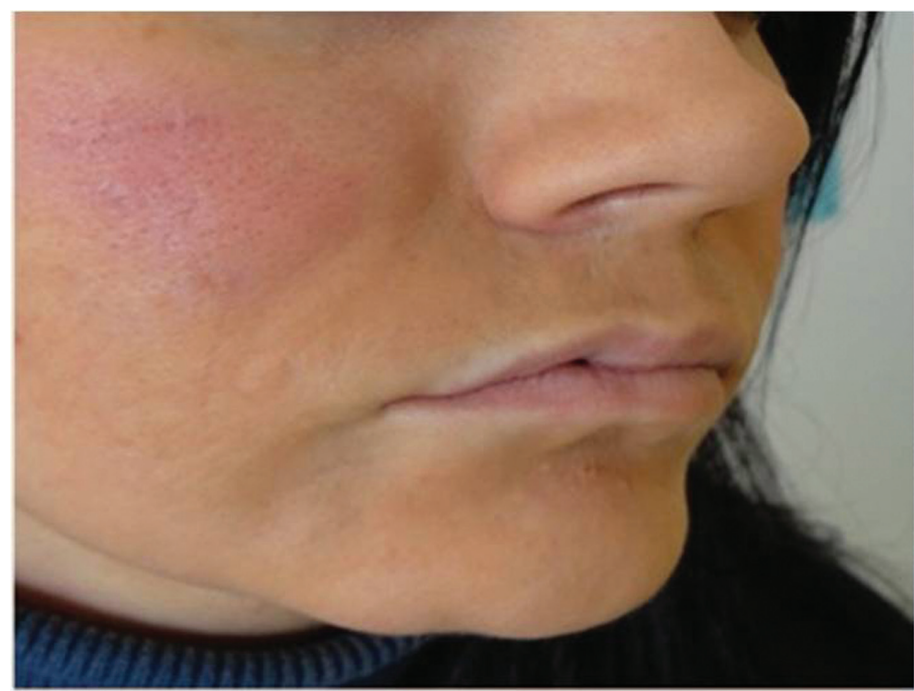

Figure 1 | Clinical aspect of Perry-Romberg syndrome before treatment.

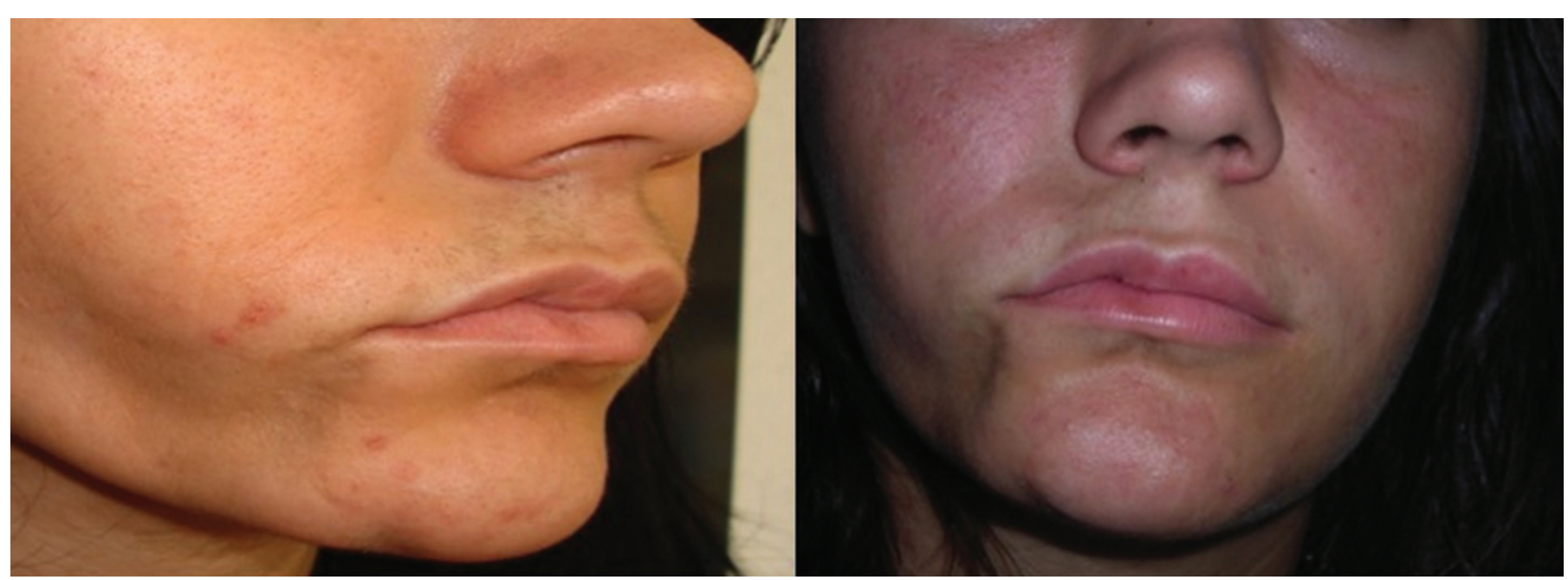

Figure 2 | Clinical aspect of Perry-Romberg Syndrome after antibiotic treatment and lipostructure.

ry-Romberg syndrome has occasionally been reported, the role of Borrelia burgdorferi sensu lato in the development of ParryRomberg syndrome is uncertain (2, 8-14). In a retrospective study on 12 patients with progressive facial atrophy, Sommer et al. (14) reported no association of both disorders. In contrast, our case indicates that there is strong evidence that Borrelia burgdorferi sensu lato infection is related to Parry-Romberg syndrome. Progressive facial hemiatrophy began when the woman was only 11 years old. She recalled a previous history of an annular lesion, likely an erythema chronicum migrans, that appeared after a tick bite. No information is available on whether antibiotic treatment was offered. Her serology for Lyme disease was positive, although only for IgM. OspC and p39 are proteins highly specific for Borrelia burgdorferi sensu lato. $\mathrm{P} 41$ is specific for the various Borrelia species of the same genus. Borrelia burgdorferi sensu lato-specific gene segments by real-time blood PCR were detected (using a locally developed method that amplifies a fragment of flagellin gene). The decrease in the CD57 lymphocyte subset may be a marker of Lyme disease (15). After the antibiotic therapy, we noticed an interruption of progression of the disease.

All these data indicate a probable Borrelia burgdorferi sensu lato infection. One could argue that the presence of IgM is significant for an early infection, but it is well known that both IgM and IgG Borrelia-specific antibodies may persist for years in some patients, which makes it impossible to distinguish between past and newly acquired infections based on seropositivity alone (16). Antibodies against Borrelia burgdorferi sensu lato in the IgM class

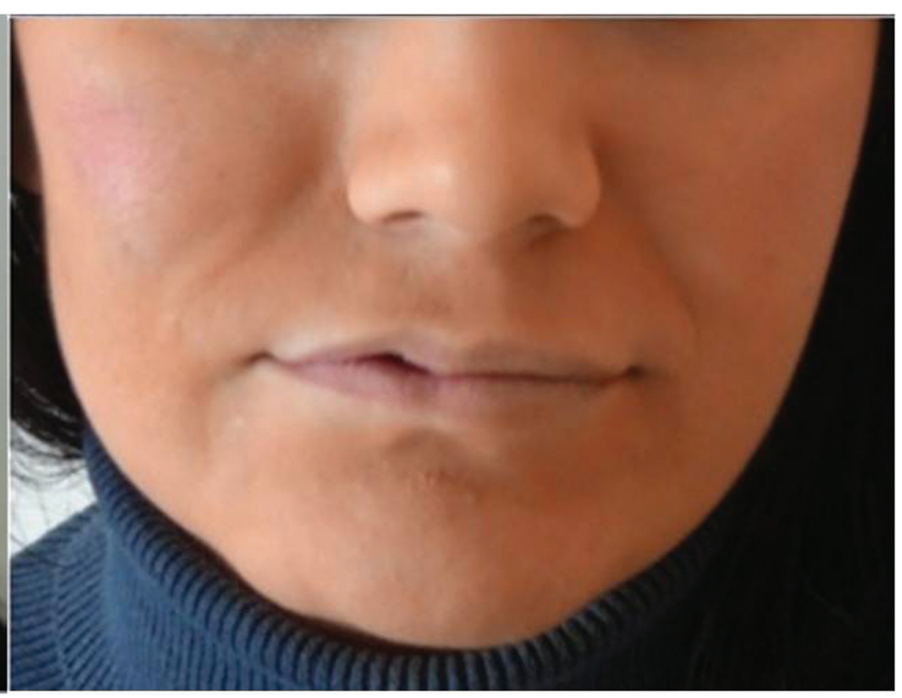


were found in another report in one of two patients with ParryRomberg syndrome (13). There are many factors (known and unknown) that affect IgM positivity and can interfere with the test and consequently the test results. A prolonged and isolated IgM response is often detected, even years later in a subset of patients. This may certainly be due to a false-positive test. On the other hand, continual IgM production with no isotype switching to IgG was postulated in patients with active disease. In our patient, we noticed a progressive decrease in the IgM titer during follow-up after antibiotic therapy.

\section{References}

1. Madasamy R, Jayanandan M, Revathy Adhavan U, Gopalakrishnan S, Mahendra L. Parry Romberg syndrome: A case report and discussion. J Oral Maxillofac Pathol. 2012;16:406-10.

2. Sahin MT, Bariş S, Karaman A. Parry-Romberg syndrome: a possible association with borreliosis. J Eur Acad Dermatol Venereol. 2004;18:204-7.

3. Kaliyadan F, Biswas K, Dharmaratnam AD. Progressive facial hemiatrophy-a case series. Indian J Dermatol. 2011;56:557-60

4. Verma R, Ram H, Gupta M, Vidhate MR. A case of extensive left-sided facial atrophy of Romberg. Natl J Maxillofac Surg. 2013;4:77-80.

5. Trevisan G, Rees DH, Stinco G. Borrelia burgdorferi and localized scleroderma. Clin Dermatol. 1994;12:475-9.

6. Gubertini N, Bonin S, Trevisan G. Lichen sclerosus et atrophicans, scleroderma en coup de sabre and Lyme borreliosis. Dermatol Reports. 2011;3:e27. doi: 10.4081/dr.2011.e27.

7. Stinco G, Trevisan G, Martina Patriarca M, Ruscio M, Di Meo N, Patrone P. Acrodermatitis chronica atrophicans of the face: a case report and a brief review of the literature. Acta Dermatovenereol Croat. 2014;22:205-8.

8. Abele DC, Bedingfield RB, Chandler FW, Given KS. Progressive facial hemiatrophy (Parry-Romberg syndrome) and borreliosis. J Am Acad Dermatol. 1990;22:531-3.

9. Stern HS, Elliott LF, Beegle PH Jr. Progressive hemifacial atrophy associated with Lyme disease. Plast Reconstr Surg. 1992;90:479-83.
It is also possible that two separate disease states coexisted coincidentally in this patient, with no cause-and-effect relationship. Some authors support this hypothesis $(13,14)$, but it is remarkable that several reports from different parts of the world show cases of Parry-Romberg syndrome associated with Lyme disease. We cannot exclude a coincidence; however, the bacteriological and serological evidence and furthermore the interruption of the disease progression after the antibiotic therapy are difficult to ignore without claiming that this association is at least suggestive. All other cases not correlated with Lyme disease still remain to be

10. Salpietro DC, Merlino MV, Bruglia S, Guarneri F, Vaccaro M. Linear scleroderma "en coup de sabre" associated with facial atrophy in a patient seropositive for Borrelia burgdorferi: a true case of molecular mimicry? Pediatr Allergy Immunol. 2004;15:570-2.

11. Baskan EB, Kaçar SD, Turan A, Saricaoglu H, Tunali S, Adim SB. Parry-Romberg syndrome associated with borreliosis: could photochemotherapy halt the progression of the disease? Photodermatol Photoimmunol Photomed. 2006;22: 259-61.

12. El-Kehdy J, Abbas 0 , Rubeiz N. A review of Parry-Romberg syndrome. J Am Acad Dermatol. 2012;67:769-84.

13. Bergler-Czop B, Lis-Swiety A, Brzezińska-Wcisło L. Scleroderma linearis: hemiatrophia faciei progressive (Parry-Romberg syndrome) without any changes in CNS and linear scleroderma "en coup de sabre" with CNS tumor. BMC Neurol. 2009;9:39.

14. Sommer A, Gambichler T, Bachrach-Buhles M, von Rothenburg T, Altmeyer $\mathrm{P}$, Kreuter A. Clinical and serological characteristics of progressive facial hemiatrophy: a case of 12 patients. J Am Acad Dermatol. 2006;54:227-33.

15. Stricker RB, Winger EE. Decreased $\mathrm{CD}_{57}$ lymphocyte subset in patients with chronic Lyme disease. Immunol Lett. 2001;76:43-8.

16. Borchers AT, Keen CL, Huntley AC, Gershwin ME. Lyme disease: A rigorous review of diagnostic criteria and treatment. J Autoimmun. 2015;57C:82-115. 